\title{
Assessing Treatment of Pruritic Scalp
}

\author{
Craig G. Burkhart ${ }^{*}, 1,2$ and Craig N. Burkhart ${ }^{3}$ \\ ${ }^{I}$ University of Toledo College of Medicine, Toledo, Ohio, USA \\ ${ }^{2}$ Ohio University College of Osteopathic Medicine, 5600 Monroe Street, Suite 106B, Sylvania, Ohio 43560, USA \\ ${ }^{3}$ Department of Dermatology, Northwestern University College of Medicine Chicago, Illinois, USA
}

\begin{abstract}
Pruritic scalp has not traditionally been specifically categorized as a separate dermatologic condition; but rather it has been included within the broad category of seborrheic dermatitis. This has severely limited testing and understanding of this entity.

The objective of this study was to assess patient satisfaction with various topical and oral therapies for pruritic scalp. For this purpose, an open-label, five-week study, 12 patients with pruritic scalp were given five different therapies, each to be used for one week. The participants then completed a survey to assess their opinion on the different therapies.

No one treatment was universally preferred. Lanacaine solution and steroid solution achieved slightly higher marks. In a few cases, oral antihistamines proved curative.

In short, pruritic scalp is a distinct condition in which one experiences episodic itching of the head without any visible or pathological sign of psoriasis, head lice, or any other scalp disorder. The divergent responses in this study underlines that there are probably different factors and trigger points which elicit the condition. More studies on this entity are warranted.
\end{abstract}

Pruritus is defined as sensations which provoke the desire to scratch. If there is no visible sign for the symptoms, one can use the term essential pruritus, pruritus sine material, or generalized pruritus [1]. However, such a designation discredits patients who have an itch secondary to metabolic alterations, systemic disease, or defect in the cutaneous neuroanatomy and neurophysiology.

On this latter point, the anatomical framework for nerve conduction includes cutaneous elicitation of itch via pruritoceptive nerve fibers and their transmitters, spinal processing of the signal, and spino-thalamico-cortal transmission of the acknowledgement of the sensation. Nerve endings and neurotransmitterss can be primary causes of pruritus. The induction of pruritus with proteinases and cytokines demonstrates the close connections between immune and neurotrophic factors in the pathophysiology of pruritus.

The scalp appears to process itch sensations slightly differently than other skin locations. The sensitivity of human scalp skin to pruritic stimuli by intradermal microdialysis in vivo is altered due to a lower innervation density of pruritic nociceptors and different central processing of itching [2]. Of note, atopic dermatitis also has been shown to have an alteration in histamine-sensitive nerve fibers [3]. Additionally, the scalp also has been shown to have an increased density of lymphatic vessels when compared to glabrous skin [4]. The lower venous and filtration pressure of the scalp leads to a diminished transcapillary protein extravasation [5]. A reduced vascular and sensory sensitivity of scalp skin to

*Address correspondence to this author at 5600 Monroe Street, Suite 106B, Sylvania, Ohio 43560, USA; Tel: 419-885-3403; Fax: 419-885-3401;

E-mail: cgbakb@aol.com pruritic agents has been established [2]. Patients with dandruff have been shown to have decreased levels of intercellular lipids in the scalp stratum corneum and impaired barrier function [6].

We propose that pruritic scalp be designated a distinct condition in which one experiences episodic itching of the head without any visible or pathological sign of psoriasis, head lice, or any other visible cause of irritation. Textbooks place this condition within the broad category of seborrheic dermatitis which classically present with dandruff and greasy scales of the hairline and eyebrows. This pilot study was performed to assess patients' responses to various topical therapies in cases of pruritic scalp.

\section{STUDY DESIGN}

A five-week, open-label study was designed to examine patient satisfaction with several topical remedies for pruritic scalp. Twelve patients, ranging in age from 32 to 66, five of whom were male, and demonstrating chronic itch of the scalp for at least four months in duration were recruited from September 2005 through May 2006 to participate in the study. The patients selected had no history of atopy, such as allergic rhinitis, asthma, hay fever, food or inhalant allergies. Additionally the patients did not have psoriasis, head lice, or demonstrable dandruff. Study subjects were also excluded if they had extensive dermatitis, or if they had, within the prior month, used any topical prescription medication for the scalp condition.

All patients were given sufficient quantities of several topical agents including a steroid solution (namely Locoid by Ferndale Laboratories), calciptriene solution (namely Dovonex by Bristol-Myers Squibb Company), benedryl solution, and lanacaine solution. Each product was to be used separately by itself for a time period of one week. They were 
also provided with an oral antihistamine, namely fexofenodine (Allegra by Sanofi Aventis) $60 \mathrm{mg}$ twice daily, to be taken for the last week without any other topical therapy. During the duration of the study, they were instructed to wash their hair daily if Caucasian, and at least twice a week if Black.

Participants were seen at baseline and at five weeks. On the return visit, survey questions were posed to the patients as presented in Table 1. Utilizing a 10-point scale, patients were asked to rate their responses to several clinical parameters. separate entity for pruritic scalp. Elie et al. [7] coined the entity 'erythematous squamous dermatoses of the scalp' for their clinical studies. Draelos discussed the frequency of patients who present at dermatological offices with scalp pruritus, but considered it a mild form of seborrheic dermatitis [8]. It is likely that this condition can be defined similarly to contact irritant dermatitis by its four main components, namely skin barrier disruption, epidermal skin changes, cytokine release, and nerve-ending changes [9]. The divergent responses in this study underlines that there are probably different factors and trigger points in this condition. When seeking better topical anti-itch therapies for

Table 1. Survey Questions Regarding Pruritic Scalp

1. Do you find washing your hair frequently helpful to reduce itching (grade on a scale of $1-10$, with 1 meaning that frequent washing did not help at all, and 10 meaning that frequent washing helped a lot)?
2
3
45
6
7
8
9
10

2. Did you find that eliminating caffeine (coffee, Coke, chocolate) reduced the itch (grade on a scale of $1-10$, with 1 meaning that eliminating caffeine did not help at all, and 10 meaning that eliminating caffeine helped a lot)?
2
3
4
5
67
89
10

3. Do you believe that stress (especially emotional stress) makes the itching worse (grade on a scale of 1 -10, with 1 meaning that stress does not affect the condition at all, and 10 meaning that stress has a major influence on the severityof the condition)?

$$
\begin{array}{llllllllll}
1 & 2 & 3 & 4 & 5 & 6 & 7 & 8 & 9 & 10
\end{array}
$$

For the next set of questions, grade on a scale of $1-10$, with 1 meaning that the product did not help at all, and 10 meaning that the product was fantastic.

4. Did you find locoid solution helped reduce the itching of your scalp?

$$
\begin{array}{llllllllll}
1 & 2 & 3 & 4 & 5 & 6 & 7 & 8 & 9 & 10
\end{array}
$$

5. Did you find dovonex solution helped reduce the itching of your scalp?

$$
\begin{array}{llllllllll}
1 & 2 & 3 & 4 & 5 & 6 & 7 & 8 & 9 & 10
\end{array}
$$

6. Did you find benedryl solution helped reduce the itching of your scalp?

$$
\begin{array}{llllllllll}
1 & 2 & 3 & 4 & 5 & 6 & 7 & 8 & 9 & 10
\end{array}
$$

7. Did you find lanacane solution helped reduce the itching of your scalp?

$\begin{array}{llllllllll}1 & 2 & 3 & 4 & 5 & 6 & 7 & 8 & 9 & 10\end{array}$

8. Did you find the oral antihistamine helped reduce the itching of your scalp?

$\begin{array}{llllllllll}1 & 2 & 3 & 4 & 5 & 6 & 7 & 8 & 9 & 10\end{array}$

\section{RESULTS}

Table 2 shows the survey tabulations with the averages displayed from the twelve questionnaires obtained. In this small study, the only query which was distinctly affirmative was that frequent hair washing reduced scalp pruritus. Not one product received high accolades from all participants. Use of lanacane solution and steroid solution were slightly preferred for topical care. The two queries with the most divergence were whether antihistamines helped and whether there is an emotional component to the itch. Two of the twelve participants had excellent relief with oral antihistamine therapy.

\section{CONCLUSIONS}

Pruritic scalp has not traditionally been specifically categorized as a separate dermatologic condition, and this has severely limited our understanding of this entity. Although seborrheic dermatitis is well represented in the literature, only a few authors have even suggested the existence of a the scalp, one must first screen for causative factors, such as viral, fungal, or parasitic elements. Thereafter, symptomatic therapy includes substituting some other sensation by cooling, heating, and/or counterirritation, by anesthesia of sensory nerve endings with local anesthetics, blocking mediators of pruritus to deplete substance $\mathrm{P}$ or to block acetylchoine release, and reducing inflammation of the skin with corticosteroids or immunomodulators. This report is a pilot study in the hopes of finding better therapies for afflicted patients. From our observations, a starting treatment for afflicted patients may include topical steroid and lanacaine in addition to a trial with oral antihistamines.

Pharmaceutical companies have offered several studies on seborrheic dermatitis [8, 10-15], an entity which seems to encompass all scalp dermatoses save for psoriasis. An association between seborrheic dermatitis and the lipophilic yeast that reside in the hair follicles has been attempted; however there is no simple stoichiometric relationship between yeast numbers and severity of the condition [16]. In short, Malassezia would not seem to play a significant etiologic 
role in pathogenesis, and should be considered a secondary effect [17]. Only in a few reports is the symptom of pruritus been considered as part of the disease process [8, 13]. More specific definitions of clinical states of the scalp should improve our understanding of conditions such as pruritic scalp.

Table 2. Averages from Patient Assessment of Clinical Parameters on the 10-Scale Used in the Survey

\begin{tabular}{|l|c|}
\hline \multicolumn{1}{|c|}{ Query } & $\begin{array}{c}\text { Average } \\
\text { Score }\end{array}$ \\
\hline \hline Did washing the hair reduce itching? & 8.3 \\
\hline Did eliminating caffeine reduce the itch? & 1.6 \\
\hline Does emotional stress make the itching worse? & 6.2 \\
\hline How well did locoid solution reduce the itching of your scalp? & 5.3 \\
\hline How well did dovonex solution reduce the itching of your scalp? & 4.1 \\
\hline How well did benedryl solution reduce the itching of your scalp? & 4.0 \\
\hline How well did lanacane solution reduce the itching of your scalp? & 5.5 \\
\hline How well did oral antihistamine reduce the itching of your scalp? & 5.3 \\
\hline
\end{tabular}

Caffeine intake has been shown to increase serotonin levels and has been associated with exacerbations of some disease states [18-21]. This dietary elimination did not affect pruritus in this patient study of pruritic scalp.

Some reports suggest that stress, and especially emotional stress, makes scalp itching exacerbate. The itching appears to be a response to thwarted urges, frustration, or just a fidgety behavior [22]. There was definitely not a concensus on this question from patient responses. Similar to previous classification of atopic dermatitis as neurodermatitis in previous generations [23, 24], stress factors are not the major impetus of scalp pruritus, and research in this area will be thwarted until such assumptions are dispelled.

There are several caveats to this study. Product preferences may have been related to base characteristics, unrelated to active ingredients. Also, this trial was of short duration with small numbers of patients, and the findings cannot adequately inform practitioners regarding the use of topical remedies for this entity.

This pilot study with twelve patients does not clarify the clinical uncertainty regarding the most effective treatment for pruritic scalp. Trials of short duration with small numbers of patients do not adequately inform practitioners regarding the use of these topical remedies. However, it does bring attention to a distinct entity, namely pruritic scalp, which does warrant further investigation.

\section{REFERENCES}

[1] Bernhard JD. Itch and pruritus: what are they, and how should itches be classified? Dermatol Ther 2005; 18: 288-91.
[2] Ruwied R, Zeck S, Schmelz M, McGlone F. Sensitivity of human scalp skin to pruritic stimuli investigated by intradermal microdialysis in vivo. J Am Acad Dermatol 2002; 47: 245-50.

[3] Wahlgren $\mathrm{CF}$, Hagermarek O, Bergstrom R. The antipruritic effect of a sedative and a non-sedative antihistamine in atopic dermatitis. Br J Dermatol 1990; 122: 545-51.

[4] Lubach D, Ludemann W, Berens VR. Recent findings on the angioarchitecture of the lymph vessel system of human skin. Br J Dermatol 1996; 135: 733-7.

[5] Reed RK. Transcapillary albumin extravasation in rat skin and skeletal muscle: effect of increased venous pressure. Acta Physiol Scand 1988; 134: 375-82.

[6] Harding CR, Moore AE, Rogers JS, Meldrum H, Scott AE, McGlone RP. Dandruff: a condition characterized by decreased levels of intercellular lipids in scalp stratum corneum and impaired barrier function. Arch Dermatol Res 2002; 294: 221-30.

[7] Elie R,Durocher LP, Koavalec EC. Effect of salicylic acid on the activity of betamethasone-17,21-dipropionate in the treatment of erythematous squamous dermatoses. J Int Med Res 1983; 11: 108 12 .

[8] Draelos ZD. An evaluation of topical 3\% salicylic acid and 1\% hydrocortisone in the manintenance of scalp pruritus. J Cosmet Dermatol 2005; 4: 193-7.

[9] Burkhart CG, Burkhart HR. Contact irritant dermatitis and antipruritic agents: the need to address the itch. J Drugs Dermatol 2003; 2 : 143-6.

[10] Hersle K, Mobacken H, Nordin P. Mometasone furoate solution $0.1 \%$ compared with keroconazole shampoo $2 \%$ for seborrheic dermatitis of the scalp. Curr Ther Res 1996; 57: 516-22.

[11] Rigopoulous D, Ioannides D, Kalogeromitros D, Gregoriou S, Katsambas A. Pimecrolimus cream $1 \%$ vs betamethasone 17 valerate $0.1 \%$ cream in the treatment of seborrheic dermatitis. A randomized open-label clinical trial. Br J Dermatol 2004; 151: 1071-5.

[12] Gupta AK, Nicol KA. Ciclopirox 1\% shampoo for the treatment of seborrheic dermatitis. Int J Dermatol 2006; 45: 66-9.

[13] Michel V, Reygagne P, Dupuy P. Results of a clinical study comparing $1 \%$ icthyol and $4 \%$ coaltar in seborrheic dermatitis of the scalp. J Europ Acad Dermatol Vener 1997; 9(Suppl 1): S186.

[14] Aly R, Katz HI, Kempers SE, et al. Ciclopirox gel for seborrheic dermatitis of the scalp. Int J Dermatol 2003; 42: S19-22.

[15] Aron-Brunetiere R, Dompmartin-Pernot D, Drouhet E. Treatment of pityriasis capitis (dandruff) with econazole nitrate. Acta Dermato-Vener 1977; 57: 77-80.

[16] Gupta AK, Kohli Y, Summerbell RC. Quantitative culture of Malassezia species from different body sites of individuals with or without dermatoses. Med Mycol 2001; 39: 243-51.

[17] Burkhart CG, Burkhart CN. Therapeutic implications of chemically- and biologically-altered sebum being one cause of seborrheic dermatitis. Open Dermatol J 2008; 2: 1-4. http://www.bentham. org/open/todj/openaccess2.htm

[18] Cederberg J, Knight S, Svenson S, Melhus H. Itch and skin rash from chocolate during fluoxetine and sertraline treatment: case report. BMC Psych 2004; 4: 36-41.

[19] Friend WG. The cause and treatment of idiopathic pruritus ani. Dis Colon Rectum 1977; 20: 40-2

[20] Goldman D. Tracrolimus ointment for the treatment of steroidinduced rosacea: a preliminary report. J Am Acad Dermatol 2001; 44: 995-8.

[21] Settipane GA. The restaurant syndromes. N Engl Reg All Proceed 1987; 8: 39-46.

[22] Adler HM. Might a psychosocial approach improve our understanding of itching and scratching? Int J Dermatol 2003; 42: 160-3. Johnson WR (ed.). On Cutaneous Diseases. London: London Press, 1808.

[24] Von Hebra H. Dermatomycosis Diffusa Flexorum, in die Krankhaften Veranderungen der Haut and Ihrer Anhangsgebilde Mit Ihrer Beziehungen $\mathrm{Zu}$ der Krankheiten Des Gesamtoranismus. Braunschweig: Wreden, 1884 